\title{
a-(1 $\rightarrow 4)$-linked D-galacturonic Acid Based Linear Homopolymer as Drug Release Modulator in HPMC Based Hydrodynamically Balanced System
}

\author{
Amit Verma ${ }^{1,2}$, Neetu Sachan ${ }^{1}$, Kamla Pathak $^{3}$, Anurag Verma ${ }^{1, *}$ \\ 'Department of Pharmaceutics, IFTM University, Moradabad, Uttar Pradesh, INDIA. \\ 2Department of Pharmacy, MJP Rohilkhand University, Bareilly, Uttar Pradesh, INDIA. \\ ${ }^{3}$ Faculty of Pharmacy, Uttar Pradesh University of Medical Sciences, Saifai, Etawah, Uttar Pradesh, INDIA.
}

\begin{abstract}
Objectives: Natural polysaccharides are increasingly being used for diverse applications in drug delivery such as controlled release of drugs owing to their wide availability, renewability, biodegradability and biosafety. Furthermore, polysaccharides from plants utilized as food may possibly mitigate regulatory requirement for approval as well. Mung bean (Vigna radiata L. seeds) is a key cereal in India composed of several non-starch polysaccharides. In the present work we have attempted to isolate, characterize and pharmaceutically evaluate the polysaccharide derived from dehusked Mung beans using water-acetone precipitation method. Methods: The obtained polysaccharide was characterized using Fourier Transform Infrared Spectroscopy, Differential Scanning Calorimetry, Powder X-ray diffraction and ${ }^{13} \mathrm{C}$ Nuclear Magnetic Resonance spectroscopy. Further, the polysaccharide derived from Mung beans was carboxymethylated using Williamson Ether Synthesis. The degree of substitution of carboxymethylated Mung bean polysaccharide was determined to be 0.76 . The carboxymethylated polysaccharide was then used in combination with hydroxypropyl methylcellulose K4M to fabricate hydrodynamically balanced sustained release capsule dosage form taking moxifloxacin $\mathrm{HCl}$ as model drug. Results: The system emerged as
\end{abstract}

a hydrodynamically balanced system that remains floated for $12 \mathrm{hr}$. The drug release mechanisms involved swelling, dissolution and erosion of polymer matrices. The erosion operated at later times and was sensitive to the osmotic stress exerted by ionic polymer and the drug present in the matrix. Conclusion: The findings of the study suggest that polymer matrices composed of chemically modified Mung bean polysaccharide and hydroxypropyl methylcellulose $\mathrm{K} 4 \mathrm{M}$ may form a promising carrier for sustained stomach specific delivery of model drug moxifloxacin Hydrochloride.

Key words: Mung bean polysaccharide, Williamson ether synthesis, Carboxymethylated polysaccharide, Moxifloxacin Hydrochloride, Hydrodynamically balanced system.

\section{Correspondence}

\section{Prof. Anurag Verma}

Department of Pharmaceutics, IFTM University, Moradabad 244001, Uttar Pradesh, INDIA.

Email: anuragvermaiftm@yahoo.co.in

DOI: 10.5330/ijpi.2020.4.86

\section{INTRODUCTION}

Recently, the evolution and utilization of polymers derived from plant sources have garnered considerable interest due to their wide availability, renewability, biodegradability and biosafety. Moreover, derivation of polysaccharides from natural sources such as pulses/cereals may possibly mitigate regulatory requirement for approval as well. ${ }^{1}$ Because of this, plant derived polysaccharides are increasingly being used for distinct applications in drug delivery. ${ }^{2}$ The seeds of Vigna radiate L. are an important pulse consumed predominantly in India and other Asian countries including China, Burma, Korea, Pakistan, Japan, Thailand and other parts of Southeast Asia. Mung bean is composed of several non-starch polysaccharides. Most of the reported polysaccharides from Vigna radiate L. seeds were derived from water extracts. The derived polysaccharides have been evaluated for antioxidant and immunoregulatory activities ${ }^{3-5}$ but not for pharmaceutical drug delivery applications. In the present work the authors have made an attempt to isolate, characterize and pharmaceutically evaluate the polysaccharide derived from dehusked Mung beans using water-acetone precipitation ${ }^{6,7}$ method. The obtained polysaccharide was studied utilizing techniques such as Fourier Transform Infrared Spectroscopy (FTIR), Differential Scanning Calorimetry (DSC), Powder X-ray diffraction (PXRD) and ${ }^{13} \mathrm{C}$ NMR spectroscopy. Further, the polysaccharide derived from Mung bean was chemically modified using Williamson Ether Synthesis. The carboxymethylated polysaccharide was then used to fabricate single unit hydrodynamically balanced (HBS) system taking moxifloxacin $\mathrm{HCl}$ as hydrophilic model drug.

Among the reasons for selecting moxifloxacin $\mathrm{HCl}(\mathrm{MX})$ is its utility in the treatment and prevention of upper gastrointestinal tract infections. MX has been reported to present in high tissue concentration in the stomach mucosa $(10.90 \pm 5.10 \mathrm{mg} / \mathrm{kg})$; which was up to 9.7 times superior to the serum concentrations. ${ }^{8}$ This increased concentration of MX in the stomach mucosa was another reason for potential use of MX in the treatment of bacterial infections in the upper gastrointestinal tract. Therefore, in the present investigation, MX was considered as a model drug for the stomach specific drug delivery through HBS capsules. Another reason for selecting the MX as model drug was its hydrophilicity $(24 \mathrm{mg} / \mathrm{ml})$. It has been reported that drugs with high aqueous solubility created high diffusional driving force coupled with osmotic stress in hydrophilic polymer matrices leading to burst release of drugs. ${ }^{9,10}$

\section{MATERIALS AND METHODS}

\section{Materials}

Dehusked and dehulled Mung beans (Tata Sampann Mung Dal Split) were purchased from local market. Chloroacetic acid, Sodium 
hydroxide, Ethanol (95\%) and concentrated Hydrochloric acid (36.5$38 \%$ ) were of laboratory reagent grade (Central Drug House., India). Hydrochloric acid $(\mathrm{HCl})$ reagent ACS (30\%, ultrapure) was obtained from Spectrum Chemical, India. Acetone was purchased from Qualigens, India. All the reagents were used procured as such. Deionized water used was of Milli-Q grade, generated in our lab.

\section{Isolation of polysaccharide}

Dehusked Vigna radiata L. seeds were washed with distilled water until the washing was completely free from any haziness. Thereafter, the seeds were soaked in deionized water (1:3, seed: water ratio) for $24 \mathrm{~h}$. With the help of a muslin cloth, the seeds were removed and filtrate was collected in a glass borosilicate glass beaker. The filtrate was then treated with acetone (1:4, filtrate: acetone ratio) as precipitating agent for polysaccharide. ${ }^{6,7}$ The extracted polysaccharide was subjected to three washings of $20 \mathrm{ml}$ each with Milli-Q grade Deionized water. The obtained product was dried in oven at $50^{\circ} \mathrm{C}$ for $12 \mathrm{~h}$. The dried product was finally powdered and passed through sieve no. 20 and stored in a desiccator until further use.

\section{Characterization of Mung Bean Polysaccharide}

\section{Solubility Analysis}

The solubility of Mung Bean Polysaccharide (MBP) was determined in distilled water, $0.1 \mathrm{M} \mathrm{HCl}$, mixed phosphate buffer, $\mathrm{pH} 6.8$, Dimethylsulfoxide (DMSO) and Tetrahydrofuran. For this, $0.1 \mathrm{~g}$ of MBP was dispersed in $10 \mathrm{ml}$ of individual solvents in a glass beaker $(25 \mathrm{ml}$ capacity) at $25^{\circ} \mathrm{C}$ and $37^{\circ} \mathrm{C}$. The contents of the beaker were than stirred using a magnetic stirrer for $1 \mathrm{hr}$ and then kept overnight. The contents were filtered and the drug was assayed at $290 \mathrm{~nm}$ in the filtrate.

\section{Spectroscopic analysis}

\section{Fourier Transform Infrared characterization}

The Fourier Transform infrared spectrum (FTIR) of MBP was generated using Fourier Transform Infrared Spectrophotometer (Perkin Elmer IR Version 10.6.0). The discs made up of MBP and $\mathrm{KBr}$ (2 mg MBP in 200 $\mathrm{mg} \mathrm{KBr}$ ) were scanned in the range of $400-4000 \mathrm{~cm}^{-1}$. The resolution was $2 \mathrm{~cm}^{-1}$.

\section{${ }^{13} \mathrm{C}$ Nuclear Magnetic Resonance (NMR) characterization}

The high resolution solid-state ${ }^{13} \mathrm{C} C \mathrm{CP} / \mathrm{MAS}$ NMR spectrum was recorded on the $400 \mathrm{MHz}$ Varian solid-state NMR spectrometer at IIT, Roorkee, India. Briefly, the spectrum was recorded at the resonance frequency of about $100 \mathrm{MHz}$ The spectrum was obtained at room temperature averaging over 5000-33000 scans. The chemical shifts were referenced to the Tetramethylsilane (TMS) using adamantane as an external standard.

\section{Thermal Analysis}

\section{Differential scanning Calorimetry}

Differential scanning Calorimetry (DSC) analysis of MBP (5 mg) was carried out over $50-250^{\circ} \mathrm{C}$ at an interval of $5^{\circ} \mathrm{C} /$ minute using duplicate samples in crimped aluminum pans. Indium was used for the calibration of the instrument.

\section{X-Ray Diffraction Studies}

\section{Powder X-ray diffraction characterization}

The Powder X-ray diffraction (PXRD) pattern of the MBP was recorded using copper radiation $(40 \mathrm{kV}, 30 \mathrm{~mA})$, on Philips Analytical X-RD (Model: PW 3710, Holland), in the range five to 1250 at the scanning rate of $0.020 / \mathrm{min}$ of $2 \theta$.

\section{Carboxymethylation of Mung Bean Polysaccharide}

The MBP was carboxymethylated following Williamson ether synthesis. ${ }^{11-12,12 a}$ Carboxymethylation was affected by Chloroacetic acid as reported by Kumar et al. with some modification. For Carboxymethylation, $2 \mathrm{~g}$ of MBP was dispersed separately in Milli-Q grade Deionized water $(100 \mathrm{ml})$ in a $250 \mathrm{ml}$ jacketed thermostated glass reactor equipped with a magnetic stirrer. After the MBP was dispersed in the deionized water, a known volume of sodium hydroxide solution (45\%, w/w) was added, at about $1 \mathrm{ml}$ per $15 \mathrm{~min}$, with uninterrupted stirring room temperature. An aliquot part of $15 \mathrm{ml}$ Chloroacetic acid was then added to the reaction mixture, over a period of $10 \mathrm{~min}$. The chemical reaction mixture was then heated to $70^{\circ} \mathrm{C}$ for $30 \mathrm{~min}$ with uninterrupted stirring. The reaction mixture was then cooled and suspended into $80 \%$ $\mathrm{v} / \mathrm{v}$ ethanol. The precipitated material was filtered and washed three times with $60 \mathrm{ml}$ of $80 \% \mathrm{v} / \mathrm{v}$ ethanol, dried in an oven at $60^{\circ} \mathrm{C}$ overnight. ${ }^{12}$ The carboxymethyl Mung bean polysaccharide (CMBP) was characterized by FTIR spectroscopy as described in previous sections; and subjected to other studies described below.

\section{Degree of substitution of Carboxymethyl Mung Bean Polysaccharide}

The extent of carboxymethylation of carbohydrate polymers is expressed in terms of degree of substitution. It is the average number of substituted carboxymethyl groups per anhydro sugar unit present on the carbohydrate polymers. In the present study, the degree of substitution (DS) ${ }^{13,14}$ was determined by potentiometric back titration and was calculated using the equation 1 :

$\mathrm{DS}=\frac{0.162 \mathrm{~A}}{1.162 \text { Aat }} \quad$------- (Eq.1)

Here, $\mathrm{A}$ is the mill equivalents of $\mathrm{NaOH}$ required per gram of sample.

\section{Equilibrium swelling}

Equilibrium swelling of both MBP and CMBP were determined in $\mathrm{pH}$ 1.2 and 6.8 buffers ( $\mathrm{HCl}-\mathrm{KCl}$ and mixed Phosphate buffer). A carefully pre weighed amount $(100 \mathrm{mg})$ of the MBP or CMPB $\left(\mathrm{W}_{1}\right)$, packed in bags made up of muslin cloth, was submerged in $50 \mathrm{~mL}$ each of the buffer solution maintained at $37^{\circ} \mathrm{C}$ and left to swell for $2 \mathrm{~h}$. The swollen mass was removed from the muslin cloth and the excess water was removed with the help of a tissue paper. The swollen mass was then reweighed $\left(\mathrm{W}_{2}\right)$ on an electronic balance. The swelling index was calculated by the following equation: ${ }^{15}$

Swelling index $=\frac{\mathrm{W}_{2}-\mathrm{W}_{1}}{\mathrm{~W}_{1}} \times 100$

\section{Carboxymethyl Mung Bean Polysaccharide as drug release modulator}

To study the effect of CMBP on drug release, single unit HBS capsules were fabricated by physically mixing moxifloxacin $\mathrm{HCl}$ with the required quantity of HPMC K4M (non-ionic polymer) and CMBP (anionic polymer) using laboratory double cone mixer for $15 \mathrm{~min}$ (Table 1). The blended mass was then encapsulated into hard gelatin capsules (size 0 ) using hand operated capsule filling machine.

The prepared formulations were tested for in vitro drug release and drug release kinetics using a USP XXXI dissolution apparatus (paddle type, Electro Lab, Mumbai, India) at $50 \mathrm{rpm}$ in $900 \mathrm{ml} \mathrm{HCl}-\mathrm{KCl}$ buffer, $\mathrm{pH}$ 1.2 at $37 \pm 0.5^{\circ} \mathrm{C}^{16,17}$ At predetermined intervals, a $1 \mathrm{ml}$ aliquot part was withdrawn and replenished with an equal volume of fresh $\mathrm{HCl}-\mathrm{KCl}$ buffer. The withdrawn samples were analyzed UV spectrophotometrically at $290 \mathrm{~nm}$. 


\section{RESULTS}

\section{Isolation of Mung Bean Polysaccharide}

The polysaccharide was extracted by a water extraction process with no involvement of heat treatment during the extraction process. The yield of MBP was found to be $7 \mathrm{~g} / 100 \mathrm{~g}$ of dehusked Mung bean. The extracted MBP was slight yellowish white free flowing powder.

\section{Solubility of Mung Bean Polysaccharide}

The solubility of MBP was determined in various solvents and buffers, the results are given in Table 2.

\section{Fourier Transform Infrared spectrum of Mung Bean Polysaccharide}

FTIR spectra of MBP (Figure 1) exhibited a broad stretching peak at $3404 \mathrm{~cm}^{-1}$ for $\mathrm{OH}$ stretch, intermolecular $\mathrm{H}$-bridge between the $\mathrm{OH}$ groups and a weak band at $2930 \mathrm{~cm}^{-1}$ due to $\mathrm{CH}_{2}$ antisymmetric stretch, conforming carbohydrate nature of the polymer. The bands at 1153 and $1018 \mathrm{~cm}^{-1}$ may be assigned to $\mathrm{C}-\mathrm{O}-\mathrm{C}$ and $\mathrm{C}-\mathrm{O}$ stretching vibrations of pyranose ring common to all polysaccharides. A weak peak at $1119 \mathrm{~cm}^{-1}$ could be due to ring antisymmetric stretch. FTIR band at $1244 \mathrm{~cm}^{-1}$ could be attributed to $\mathrm{OH}$ in-plane deformation in $\mathrm{COOH}$ groups. The peaks at wave number 1407 and $1454 \mathrm{~cm}^{-1}$ could be attributed to $\mathrm{CH}_{2}$ scissors vibration. The FTIR peak at $1642 \mathrm{~cm}^{-1}$ could be attributed to free carboxylate group in the MBP. The broad spectral region between $1800-700 \mathrm{~cm}^{-1}$ serves as fingerprint region for monosaccharide types, substituents and epimers of different sugars. ${ }^{24}$ In this broad spectral region, the deformation peak of C-H occurred between $1500-1200 \mathrm{~cm}^{-1}$ [1244 and $1407 \mathrm{~cm}^{-1}$ ] and that of C-O-C occurred in the range of 1150$1000 \mathrm{~cm}^{-1}\left[1018,1153 \mathrm{~cm}^{-1}\right]$.

\section{${ }^{13} \mathrm{C}$ NMR of Mung Bean Polysaccharide}

The ${ }^{13} \mathrm{C}$ Nuclear Magnetic Resonance spectrum of MBP is exhibited in the Figure 2. A resonance at $\sim 174 \mathrm{ppm}$ corresponds to the C6 region $\left(\mathrm{COO}^{-}\right)$of the polysaccharide. The resonance peak at $100.8 \mathrm{ppm}$ can be assigned to $\mathrm{C}-1$ carbon of the polysaccharide. The peak at 100.8 could be assigned to the resonance of glycosidic $\alpha$-linkage. The resonance peak at $75.13 \mathrm{ppm}$ could be attributed to the $\mathrm{C}-4$ carbons. The resonance peak at 72.69 ppm could be attributed to C-5 carbons of the MBP, whereas, the resonance peak at $71.29 \mathrm{ppm}$ may be due to C-2 and C-3 carbon. The anomeric $\mathrm{C} 1$ resonance numbers in a ${ }^{13} \mathrm{C}$ NMR spectrum tells us about the number of different sugar residues.

\section{Powder X-ray diffractogram of Mung Bean Polysaccharide}

The PXRD pattern of MBP is exhibited in Figure 3. The carbohydrate polymer sample showed peaks at approximately 19.7, 62.5, 69.14 and $88.7^{\circ}$. However, other peaks are very weak and could not be resolved.

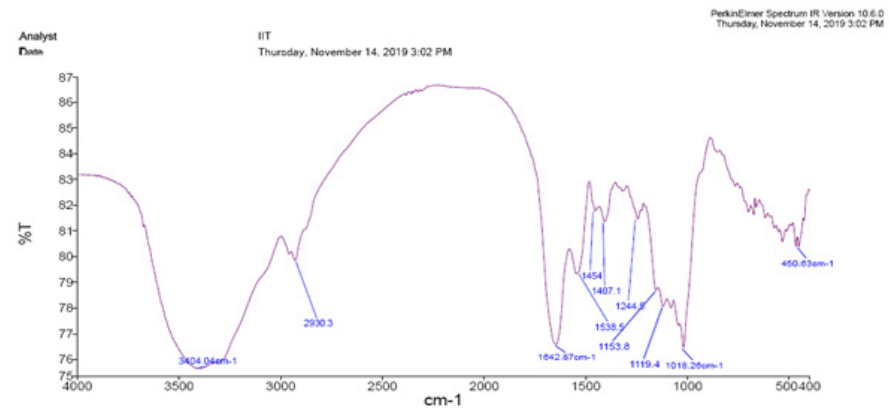

Figure 1: FTIR Spectra of Mung Bean Polysaccharide.

\section{Differential Scanning Thermogram of Mung Bean} Polysaccharide

Figure 4 shows the DSC curve of the MBP. The glass transition temperature was $127.7^{\circ} \mathrm{C}$ and there was a melting peak but unfortunately we could only see its initiation in the thermogram at around $300^{\circ} \mathrm{C}$.

\section{Carboxymethylation of Mung Bean Polysaccharide}

Carboxymethylation of MBP was done by Williamson ether synthesis. The yield of CMBP was found to be $71.88 \%$. The solubility of CPMB (1\% $\mathrm{w} / \mathrm{v}$ ) when tested in distilled water $\left(37^{\circ} \mathrm{C}\right)$, found to be partially soluble, compared to completely insoluble behavior of $\mathrm{MPB}$. The $\mathrm{pH}$ of the dispersion was found to be 6.9. It was also observed that the solution was slightly viscous; therefore, its viscosity was determined using Brookfield rheometer (LVDV-III, spindle cp 40). The viscosity was found to be 69.8 cps. The extent of carboxymethyl substitution, also called degree of substitution, of CMBP was determined to be 0.76 carboxymethyl groups/g of MBP. The CMBP was characterized by FTIR to confirm the chemical changes in CMBP.

\section{Fourier Transform Infrared Spectrum of Carboxymethyl Mung Bean Polysaccharide}

The spectra of CMBP (Figure 5) exhibited a peak due to $\mathrm{O}-\mathrm{H}$ stretching of alcoholic group at $3439 \mathrm{~cm}^{-1}$. This peak is comparatively less broad than the corresponding peak in the FTIR spectrum of MBP $\left(3439 \mathrm{~cm}^{-1}\right)$. This could be attributed to the carboxymethylation of some of the $\mathrm{OH}$ groups.

FTIR bands at $2927 \mathrm{~cm}^{-1}, 1641 \mathrm{~cm}^{-1}, 1413 \mathrm{~cm}^{-1}, 1217 \mathrm{~cm}^{-1}$ and 1018 $\mathrm{cm}^{-1}$ correspond to, $\mathrm{C}-\mathrm{H}$ stretching of the $-\mathrm{CH}_{2}$ groups, $\mathrm{C}=\mathrm{O}$ stretch of COO- of carboxylate anion, asymmetric and symmetric stretching of $\mathrm{COO}^{-}$group, stretching vibrations of $\mathrm{C}-\mathrm{O}$ in ethers and esters and stretching vibration of $\mathrm{C}-\mathrm{O} \alpha$-glycosidic bond respectively. The bands at $814,642,564$ and $525 \mathrm{~cm}^{-1}$ in the infrared spectra are attributed to the skeletal modes of the pyranose ring.

\section{Swelling behavior of Carboxymethyl Mung Bean Polysaccharide}

MBP exhibited low swellability in both acidic ( $\mathrm{pH}$ 1.2) and basic media (pH 6.8) [150.65 and 179.45\%]. CMBP exhibited higher swellability in pH 6.8 than in pH 1.2 (203.43 and 297.67\%).

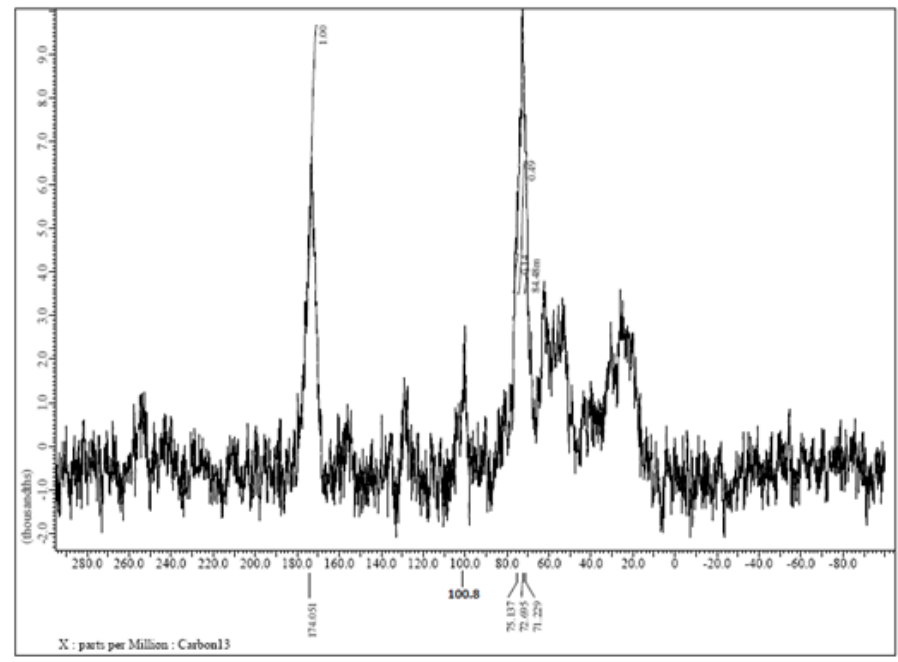

Figure 2: ${ }^{13} \mathrm{C}$ NMR Spectra of Mung Bean Polysaccharide. 
Drug release modulation by Carboxymethyl Mung Bean Polysaccharide

In the presented study, we have made an attempt to study the effect of combining CMBP with HPMC K4M on the release of a weakly basic drug $\mathrm{MX}$ in acidic dissolution medium (0.1 M HCl, $\mathrm{pH} 1.2)$. The drug release was determined in triplicate.

Various hydrophilic polymeric matrices were prepared as depicted in Table 1. After combining with MX, the drug-polymer mixtures were encapsulated in transparent size 0 capsules and subjected to in vitro drug release studies. During the entire study it was observed that all the formulations remained buoyant on the dissolution medium (no lag time) during the drug release studies and once the capsule shell completely disintegrated (30 min), the hydrated polymer matrices acquired the shape of a cylindrical plug. This cylindrical plug eroded with time but remained floated for entire duration of drug release studies. Thus the developed formulations were designated as hydrodynamically balanced systems (HBS).

In the presented work, the time required to release 25, 50, 75 and $100 \%$ of the drug was taken as the basis to express moxifloxacin $\mathrm{HCl}$ release from hydrodynamically balanced capsule dosage form (Figure 6). From formulation AV1 containing HPMCK4M about 27, 56, 81 and $97 \%$ of MX was released at the end of $2^{\text {nd }}, 5^{\text {th }}, 7^{\text {th }}$ and $10^{\text {th }} \mathrm{h}$. In formulation AV2, MBP was combined with HPMC K4M. Although MBP is insoluble in 0.1 $\mathrm{M} \mathrm{HCl}$ attributed to the presence of crystalline portions in its polymer chain. MBP swells to a certain extent in the acidic medium. Being less swellable in acidic dissolution medium, MBP contribute minimally to the swelling of HPMC based hydrophilic polymer matrix, however, it might act as pore former in the swelled hydrogel matrix. From formulation

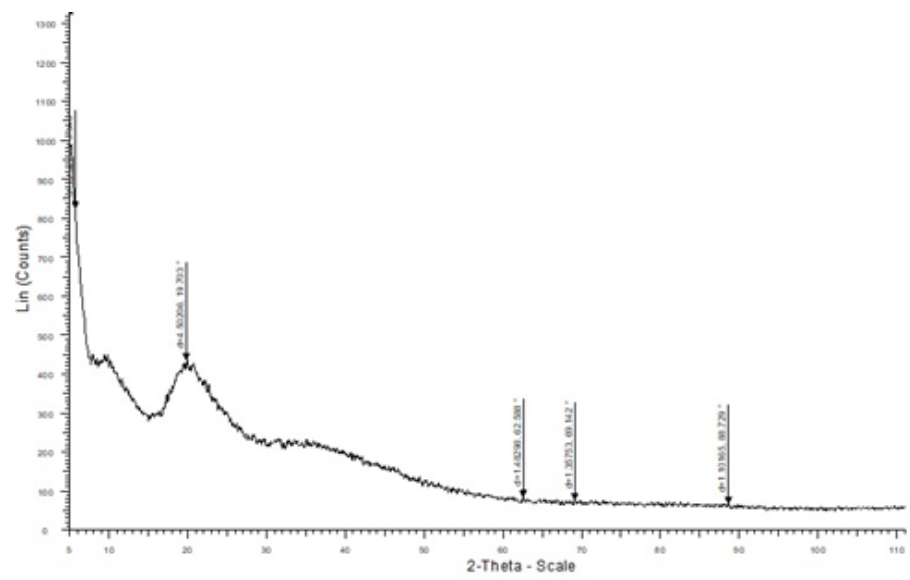

Figure 3: PXRD pattern of Mung Bean Polysaccharide.

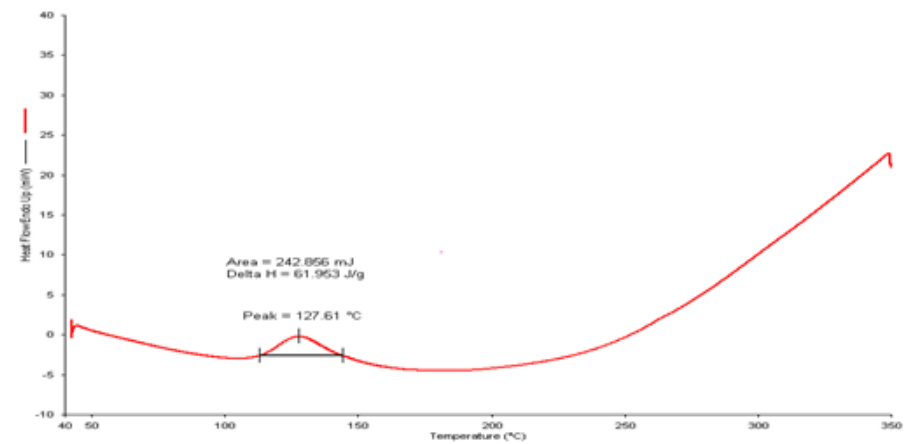

Figure 4: DSC thermogram of Mung Bean Polysaccharide.
AV2, about $24,52,82$ and $99 \%$ MX was released at the end of $1^{\text {st }}, 4^{\text {th }}, 6^{\text {th }}$ and $8^{\text {th }}$ hour. In case of formulation AV3, anionic CMBP was mixed, in comparable amount as that of MBP, with non-ionic HPMC K4M. About $24,47,74$ and $98 \%$ MX was released at the end of $3^{\text {rd }}, 7^{\text {th }}, 9^{\text {th }}$ and $12^{\text {th }}$ hour. Formulation AV4 composed of CMBP (50 mg), HPMC K4M (200 mg) and MX. Here about 28, 50, 80 and 99\% MX was released at the end of $3^{\text {rd }}, 6^{\text {th }}, 8^{\text {th }}$ and $11^{\text {th }}$ hour. The release profiles were comparable to the formulation AV3.

\section{DISCUSSION}

The MBP from the water extract was precipitated by acetone (1:4, water: acetone ratio). Acetone is relatively hydrophobic water-miscible solvent. When added to the water extract, it changes the hydrophilic/ hydrophobic nature of the original solvent, that is, water. As the solvent becomes less hydrophilic, the attractive hydrogen bonding forces within the polysaccharide chain become more prevalent and the portions of the chain tend to bond within and between the polysaccharide chains, rather than with the solvent. These intra- and intermolecular hydrogen bonds lead to agglomeration and precipitation as solvation decreases.

MBP was found to be practically insoluble in all the solvents tested for evaluation of the solubility. The poor solubility of MBP could be explained on the basis of molecular weight or degree of polymerization. ${ }^{18,19}$ Generally low molecular weight carbohydrate polymers having degree of polymerization $<15-20$ are water soluble. Highly branched polysaccharides are almost always exhibit high solubility in water. ${ }^{20}$ Solubility of carbohydrate polymers decreases with increase in the ease with which their molecules associate and with narrowness of molecular weight distribution. Carbohydrate polymers having regular, extended

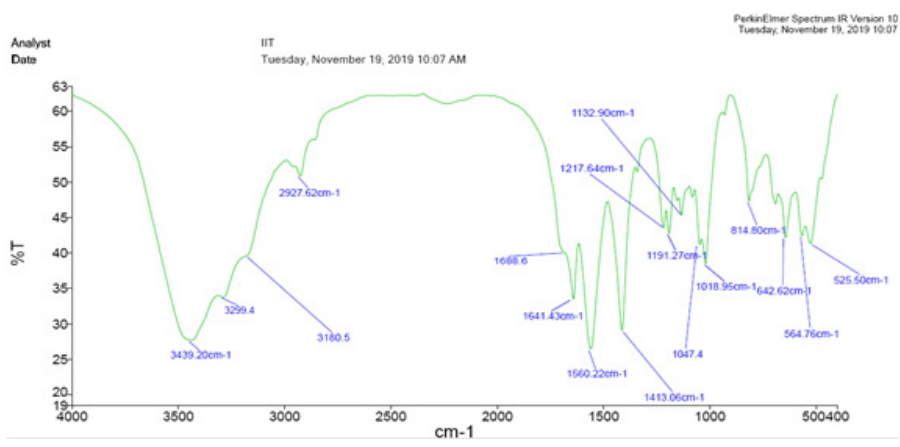

Figure 5: FTIR Spectrum of Carboxymethyl Mung Bean Polysaccharide.

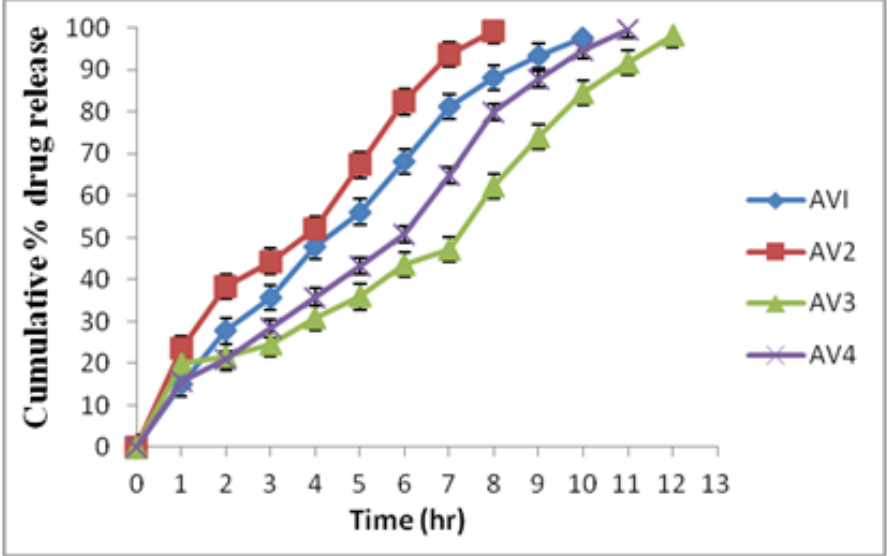

Figure 6: Cumulative \% drug release from various HBS capsule formulations $[($ Mean $\pm \operatorname{SD}(n=3)]$ 
Table 1: Composition of formulations containing moxifloxacin $\mathrm{HCl}$.

\begin{tabular}{ccccc}
$\begin{array}{c}\text { Formulation } \\
\text { code }\end{array}$ & $\begin{array}{c}\text { HPMC K4M } \\
(\mathrm{mg})\end{array}$ & $\begin{array}{c}\text { MBP } \\
(\mathrm{mg})\end{array}$ & $\begin{array}{c}\text { CMBP } \\
(\mathrm{mg})\end{array}$ & $\begin{array}{c}\text { MX } \\
(\mathrm{mg})\end{array}$ \\
\hline AV1 & 250 & ---- & --- & 218 \\
AV2 & 225 & 25 & --- & 218 \\
AV3 & 225 & --- & 25 & 218 \\
AV4 & 200 & --- & 50 & 218 \\
\hline
\end{tabular}

Table 2: Solubility of Mung Bean Polysaccharide in various solvents.

\begin{tabular}{cc}
\hline Solvents & Solubility at $25^{\circ} \mathrm{C}$ \\
\hline Distilled water & Practically insoluble \\
Acetonitrile & Practically insoluble \\
Tetrahydrofuran & Practically insoluble \\
Dimethyl Sulfoxide & Practically insoluble \\
$0.1 \mathrm{M} \mathrm{HCl}^{* *}$ & Practically insoluble (both at $25^{\circ} \mathrm{C}$ \\
& and $\left.37^{\circ} \mathrm{C}\right)$ \\
$\mathrm{pH} 6.8$ phosphate buffer & Practically insoluble(both at $25^{\circ} \mathrm{C}$ \\
& and $\left.37^{\circ} \mathrm{C}\right)$ \\
\hline
\end{tabular}

*Although insoluble but swelled in $\mathrm{pH} 6.8$ phosphate buffer

** Slightly swelled in $0.1 \mathrm{M} \mathrm{HCl}$

ribbon like structure are the ones which most easily associate and form crystals. Generally, reinforcing, cell-wall carbohydrate polymers are least soluble. Linear polysaccharides of uniform structure can develop crystalline regions which firmly tie molecular segments together in effective cross-links. Such molecules are very difficult to dissolve in water. ${ }^{21}$ It has also been reported that carbohydrate polymers composed of one type of sugar unit uniformly linked in linear chains are generally water insoluble even if the carbohydrate polymers molecules have low molecular weight with low degrees of polymerization ${ }^{16}$ (20-30). Examples of polysaccharides with least solubility include cellulose, pectic acid, alginic acid, xylan, chitin, amylose etc. All these polysaccharides have either $\alpha$ or $\beta(1 \rightarrow 4)$-glycosidic linkage. ${ }^{21}$

Upon literature survey, it was observed that the infrared spectra of carbohydrate polymers are divided into three specific Infrared regions viz. $1200-900 \mathrm{~cm}^{-1}, 3000-2700 \mathrm{~cm}^{-1}$ and $900-600 \mathrm{~cm}^{-1}$. The intensive peaks in the Infrared region between $1200-900 \mathrm{~cm}^{-1}$ are attributed to strongly coupled $\mathrm{C}-\mathrm{C}, \mathrm{C}-\mathrm{O}$ stretching and $\mathrm{C}-\mathrm{O}-\mathrm{H}, \mathrm{C}-\mathrm{O}-\mathrm{C}$ deformation modes of various oligo and polysaccharides. ${ }^{22}$ In the Infrared region 3000-2700 $\mathrm{cm}^{-1}$ carbohydrate polymers show few sharp absorption bands, for example, band at $2930 \mathrm{~cm}^{-1}$ assigned to asymmetric $\mathrm{C}-\mathrm{H}$ stretching of $\mathrm{CH}_{2}$; band at $2870 \mathrm{~cm}^{-1}$ assigned to symmetric $\mathrm{C}-\mathrm{H}$ stretching of $\mathrm{CH}_{3}$ and $\mathrm{C}-\mathrm{H}$ stretching bands in the range of $2700-3100 \mathrm{~cm}^{-1}$. The region between 900 and $600 \mathrm{~cm}^{-1}$ exhibits a number of characteristic weak bands superimposed on an underlying broad spectral contour. ${ }^{23}$ The carbohydrate polymers share a common functional group, that is, hydroxyl. These polymers self-assemble and form hydrogen-bonded network with porous hierarchy unique to respective monosaccharide building blocks. ${ }^{20}$ The FTIR peaks in the Infrared region (3600 to 3000 $\mathrm{cm}^{-1}$ ) are due to $\mathrm{O}-\mathrm{H}$ stretching modes in alcoholic groups. The stronger the hydrogen bond, the greater the intensity of the corresponding $v \mathrm{O}-\mathrm{H}$ band and the greater its shift towards lower wave numbers. The FTIR spectrum of MBP, suggested that the polysaccharide was a pyranose form of sugars.

Carbohydrate polymers generally have two NMR-active nuclei, ${ }^{13} \mathrm{C}$ and ${ }^{1} \mathrm{H}$. Chemical shifts in the ${ }^{13} \mathrm{C}$ NMR spectrum of a carbohydrate polymer follow the same general trends as for ${ }^{1} \mathrm{H}$ NMR spectroscopy, although the probability of "accidental overlap" is less because the dispersion of ${ }^{13} \mathrm{C}$ shifts ( $\delta \mathrm{C} 60-110 \mathrm{ppm}$ ) is approximately 20 times wider in the ${ }^{13} \mathrm{C}$ NMR spectrum. Compared with ${ }^{1} \mathrm{H}-\mathrm{NMR}$ spectrum; ${ }^{13} \mathrm{C}-\mathrm{NMR}$ has a wide range of displacement values and only a few signals overlap. ${ }^{25}$, ${ }^{26}$ The FTIR and ${ }^{13} \mathrm{C}$ NMR spectra of MBP, indicated it to be a pectic polysaccharide, most probably, a carbohydrate polymer molecule consisting of $\alpha-(1 \rightarrow 4)$-linked D-galacturonic acid (D-GalpA) residues.

The most important method for the structure determination of crystalline polysaccharides is powder X-ray diffraction (PXRD). The result of the XRD shows that MBP has both crystalline and amorphous portions. Structural and functional group differences in polysaccharides influence the thermal behavior and affect the transition temperature. DSC is one of the frequently used thermal techniques that estimate the exothermic or endothermic changes as temperature is increased. Most of carbohydrate polymers are composed of carboxylic acid functional groups. Thus, thermal cleavage of the carboxylate groups and generation of carbon dioxide gas from the carbohydrate backbone probably a mechanism for the thermal transitions. ${ }^{27}$ The thermal treatment in DSC resulted in physical and chemical changes in carbohydrate polymers that yield curves that are unique for a given carbohydrate polymer. $^{28}$

While the evolution and application of polymers derived from plant sources have attracted increased attention due to their sustainability, biodegradability and biosafety; they evidence certain drawbacks, such as solubility, swelling characteristics, variable chemical composition, pH-dependent solubility, poor mechanical strength. ${ }^{29-31}$ The above mentioned drawbacks of natural polymers can be overcome via chemical modification. ${ }^{32}$ The chemical modification of naturally derived polymers is the most important route to modify their properties and to use this renewable resource in the context of pharmaceutical product development. Modifications which are most frequently done to the natural polymers include carboxymethylation and grafting. Among the chemical modifications, carboxymethylation is a widely studied conversion as it increases the aqueous solubility and solution clarity of modified polymers. Site specific targeted drug release can be also achieved by attaching $\mathrm{pH}$-sensitive carboxylic acid groups to the polymeric structure, via carboxymethylation process. ${ }^{33-35}$ In the present investigation, carboxymethylation of MBP was done by Williamson ether synthesis. In this synthesis, the polysaccharide alkoxide is reacted with monocholoroacetic acid and the primary and secondary alcohol groups are substituted by carboxymethyl group by an $\mathrm{S}_{\mathrm{N}} 2$ reaction. ${ }^{36-38}$

$$
\mathrm{MBP}-\mathrm{OH}+\mathrm{NaOH}--------\rightarrow \mathrm{MBP}-\mathrm{ONa}+\mathrm{H}_{2} \mathrm{O} \text {------ (Eq. 3) }
$$$$
\text { (MBP- alkoxide) }
$$

$\mathrm{MBP}-\mathrm{ONa}+\mathrm{ClCH}_{2} \mathrm{COONa}---\rightarrow \mathrm{MBP}-\mathrm{OCH}_{2} \mathrm{COONa}+\mathrm{NaCl}--($ Eq. 4$)$ (Carboxymethylated MBP; CMBP)

CMBP exhibited $\mathrm{pH}$ dependent swelling characteristic. The $\mathrm{pH}$ dependent swellability of CMBP is attributed to the presence of carboxymethyl groups. The carboxymethyl groups ionize when the $\mathrm{pH}$ of the environment is higher than the $\mathrm{pKa}$ of the carboxylic group (3.4-3.7). Therefore, at $\mathrm{pH} \mathrm{1.2,} \mathrm{the} \mathrm{carboxyl} \mathrm{groups} \mathrm{are} \mathrm{protonated} \mathrm{to}$ promote the formation of intramolecular hydrogen bonds, thereby reducing the overall swellability. At $\mathrm{pH}$ 6.8, the carboxyl groups get deprotonated and higher swellability is observed due to collective electrostatic repulsion forces between the ionized acid groups. ${ }^{39}$ Polymer matrices composed of hydroxypropyl methyl cellulose (HPMC) with ionic/non-ionic polymers have been utilized as hydrophilic matrices to modulate the drug release and overcome most of the challenges associated with hydrophilic polymeric matrices. Hydrophilic polymer matrices made up of HPMC with anionic polymers have been reported to exhibit $\mathrm{pH}$-independent release profiles for weakly basic drugs. ${ }^{40}$ The addition 
of anionic polymers in the polymer matrix can effect drug release in alkaline dissolution media by decreasing the micro-environmental $\mathrm{pH}$ and also extend the drug release in acidic dissolution media by forming an insoluble mass, which acts as a barrier to drug diffusion. ${ }^{41}$

There was extended release of MX from formulation AV1. this could be attributed to the swelling of HPMC based polymeric matrices when exposed to acidic gelation medium, due to hydrogen bond disruption between the polymeric-chains to form a thick gelatinous layer at the boundary of the matrix, with simultaneous erosion. Further, the solubility of the drug in dissolution medium is an important factor determining the mechanism of drug release from. HPMC based hydrophilic matrices. ${ }^{42-45}$ For drugs, such as MX, that have high water solubility $(24 \mathrm{mg} / \mathrm{ml}$ in water at $25^{\circ} \mathrm{C}$ ), the drug dissolves within the gel layer and diffuses out into the dissolution media. Therefore, it is of utmost importance to ensure the integrity of the gel layer after the MX has been dissolved and released from the gel layer. ${ }^{46}$ This suggests that it is critical to have a strong gel layer through which diffusion can occur ${ }^{47}$ therefore, the extended MX release could be attributed to the high molecular weight HPMC K4M polymeric-matrices.

On the other hand there was rapid release of MX from formulation AV2. The significantly fast drug release $(p<0.05$ compared to AV1) could be attributed to the penetration of comparatively large amount of acidic dissolution medium into the hydrogel matrix due to pore formation by MBP. Consequent polymer swelling (HPMC K4M) and formation of micro cavities within the hydrated polymer matrix promoted the drug release. ${ }^{48}$ In case of formulation AV3, anionic CMBP was mixed. This has resulted in significant retardation ( $p<0.05$ compared to AV2) of MX release. This could be attributed to the synergistic increase in viscosity of the mix hydrogel, leading to increased resistance to drug diffusion. ${ }^{49}$ There could be one more reason that there is interaction between anionic CMBP and cationic MX. The release of highly soluble cationic drugs have been reported to be extended from combinations of HPMC with anionic sodium carboxymethyl cellulose compared with HPMC alone, attributed to interection between drug and anionic polymer. ${ }^{49}$

The in vitro moxifloxacin $\mathrm{HCl}$ release pattern from hydrodynamically balanced capsule formulations was studied through fitting the dissolution data into different important kinetic models. In case of hydroxypropyl methylcellulose based hydrodynamically balanced capsules bearing model drug MX, $r^{2}$ value of formulation AV1 was found best fitted to Korsmeyer-Peppas kinetic model. This model provides an insight into the kind of drug release, for the first $60 \%$ of release regardless of the geometric shape of the delivery device, mechanism occurring from the swellable polymeric-matrices. The Korsmeyer-Peppas model (equation5) is useful for the study of drug release from polymeric matrices when the release mechanism is not known or when more than one type of phenomenon of drug release is involved..$^{50,51}$

$f_{1}=\frac{M_{\mathrm{i}}}{M_{\infty}}=K t^{n} \quad$------- (Eq.5)

where $f_{1}$ is quantity of the drug released from the system; $M_{\infty}$ is the quantity of the drug at equilibrium state; $M_{i}$ is amount of the drug released over time $\mathrm{t}$; $\mathrm{K}$ is the constant of incorporation of structural modifications and geometrical characteristics of the system and $\mathrm{n}$ is the exponent of release (related to the drug release mechanism) in function of time t. Depending on the value of $n$, the diffusion of drug through polymeric matrices is either Fickian diffusion (Case I) or non-Fickian diffusion (Case II, anomalous case and super case II). Fickian diffusion defines the solute transport process in which the polymer relaxation time $\left(t_{r}\right)$ is much greater than the characteristic solvent diffusion time (td).
When $\mathrm{tr} \approx \mathrm{td}$, the macroscopic drug release becomes anomalous or nonFickian. In the Fickian diffusion, $n=0.5$ (planer film), 0.45 (cylinder) and 0.43 (sphere) and the drug release is governed by diffusion. When $0.5<$ $\mathrm{n}<1.0$, the diffusion is non-Fickian or anomalous transport (Case II) and the mechanism of drug release is governed by diffusion and swelling. The Super Case II model is characterized when $n>1$. A good-fit to the Korsmeyer- Peppas model indicates the combined effect of diffusion and relaxation mechanisms for the release..$^{50-52}$

In case of formulation $\mathrm{AV} 1$, the $\mathrm{n}$ value is 0.82 . This suggests that drug transport is non-Fickian anomalous transport. The diffusion and swelling rates have similar magnitude. The rearrangement of polymeric chains occurring slowly and the diffusion process simultaneously cause the time-dependent anomalous effects. In formulation AV2, MBP was mixed with HPMC K4M. The addition of MBP enhanced the release of MX from the hydrogel matrix, which means that insoluble MBP (20 mesh particle size) facilitated the movement of dissolution medium into the HPMC based hydrogel matrix increasing the solubilization of MX, thereby increasing the movement of drug out of the matrix. The $r^{2}$ value was found best-fit when fitted to zero-order model. The $n$ value is 0.61 . This suggests that the velocity of the dissolution medium diffusion into the interior of the polymer matrix was high and the polymeric relaxation was low compared to formulation AV1.

In case of formulation AV3, the $r^{2}$ value was found best fitted to zeroorder model. The $n$ value is 0.54 . In this case an increase in swelling of the hydrogel is expected due to addition of CMBP to the HPMC K4M matrix. The rate of polymer hydration depends on the structure of the polymer or polymer matrices used in formulations in addition to the degree of interaction of functional groups within the polymeric matrices with water. The better the interaction between a polymer surface and water, the faster the polymer hydrates because the first layer of adsorbed water molecules enables easier adsorption of additional water molecules. ${ }^{53}$ The presence of the methyl substituent of HPMC and carboxymethyl substituent of CMBP permits interaction of the molecule with water, thereby promoting hydration and consequent swelling such as that observed..$^{53}$ The magnitude of swelling was further increased with decreased polymer relaxation compared to formulation AV2 and AV1. This has allowed the erosion of the swollen matrix to occur at a rate equating to the movement of the front between the glassy and the rubbery polymer. ${ }^{49}$

In case of formulation $\mathrm{AV} 4$, the concentration of $\mathrm{CMBP}$ in the polymer matrix was increased to $50 \mathrm{mg}$. Although the drug release pattern remained anomalous but there was increase in the value of $\mathrm{n}$ (0.72 (AV4) compared to 0.61 and 0.54 for AV2 and AV3 respectively. This suggests an increase in the polymer relaxation time compared to formulation AV1 and AV2. This observation revealed that swelling, dissolution and erosion of polymer matrices were all involved in the release process. The erosion operates at later times and is sensitive to the osmotic stress exerted by ionic polymer and the drug present in the matrix.

\section{CONCLUSION}

The polysaccharide $\alpha$ - $(1 \rightarrow 4)$-linked D-galacturonic acid (D-GalpA) based linear homopolymer was successfully isolated from dehusked mung bean by cold extraction using water-acetone precipitation method. The polysaccharide was chemically modified to its carboxymethylated derivative that was used to fabricate sustained release capsules using moxifloxacin $\mathrm{HCl}$. The system emerged as a hydrodynamically balanced system that remained floated for $12 \mathrm{~h}$. Further studies are underway that would assess its in vivo performance and biosafety. 


\section{ACKNOWLEDGEMENT}

The author is grateful to the Department of Pharmaceutics, IFTM University, Moradabad, India, for support and useful guidance.

\section{CONFLICT OF INTEREST}

The authors declare no conflict of interest.

\section{ABBREVIATIONS}

MX: Moxifloxacin HCl; MBP: Mung Bean polysaccharide; CMBP: Carboxymethyl Mung Bean polysaccharide; FTIR: Fourier Transform Infrared Spectroscopy; DSC: Differential Scanning Calorimetry; PXRD: Powder X-Ray Diffraction.

\section{REFERENCES}

1. Tong $X$, Pan $W$, Su T, Zhang $M$, Dong $W$, Oi X Recent advances in natura polymer-based drug delivery systems. React Funct Polym. 2020;148:104501.

2. Karolewicz B. A review of polymers as multifunctional excipients in drug dosage form technology. Saudi Pharm J. 2016;24(5):525-36.

3. Lai F, Wen Q, Li L, Wu H, Li X. Antioxidant activities of water-soluble polysaccharide extracted from mung bean (Vigna radiata L.) hull with ultrasonic assisted treatment. Carbohydr Polym. 2010;81(2):323-9.

4. Zhong K, Lin W, Wang Q, Zhou S. Extraction and radicals scavenging activity of polysaccharides with microwave extraction from mung bean hulls. Int J Biol Macromol. 2012;51(4):612-7.

5. Yao Y, Zhu Y, Ren G. Immunoregulatory activities of polysaccharides from mung bean. Carbohydr Polym. 2016;139:61-6.

6. Moscovici M. Present and future medical applications of microbial exopolysaccharides. Front Microbiol. 2015;6:1012.

7. Kakuta M, Sone Y, Umeda T, Misaki A. Comparative structural studies on acidic heteropolysaccharides isolated from "Shirokikurage," fruit body of Tremella fuciformis Berk and the growing culture of its yeast-like cells. Agricultural Biol Chem. 1979;43(8):1659-68.

8. Wirtz M, Kleeff J, Swoboda S, Halaceli I, Geiss HK, Hoppe-Tichy T, et al. Moxifloxacin penetration into human gastrointestinal tissues. J Antimicrob Chemother. 2004;53(5):875-7.

9. AlOmari MMH, Jaafari DS, Al-Souod KA, Badwan AA. Chapter SevenMoxifloxacin Hydrochloride. Profiles of Drug Substances, Excipients and Related Methodology. 2014;39:299-331.

10. Pubchem Substance and Compound Databases. Pub Chem CID $=101526$, https://pubchem.ncbi.nlm.nih.gov/compound/101526

11. Kaity S, Ghosh A. Carboxymethylation of Locust Bean Gum: Application in Interpenetrating Polymer Network Microspheres for Controlled Drug Delivery. Ind Eng Chem. 2013;52(30):10033-45

12. Mohapatra S, Siddiqui AA, Anwar M, et al. Synthesis and characterization of novel carboxymethyl Assam Bora rice starch for the controlled release of cationic anticancer drug based on electrostatic interactions. AAPS Pharm Sci Tech. 2018;19(1):134-47. a) Ahuja M, Kumar A, Singh K. Synthesis, characterization and in vitro release behavior of carboxymethyl xanthan. Int J Biol Macromol. 2012;51(5):1086-90.

13. Lemieux M, Gosselin P, Mateescu MA. Influence of Drying Procedure and of Low Degree of Substitution on the Structural and Drug Release Properties of Carboxymethyl Starch. AAPS Pharm Sci Tech. 2010;11(2):775-85

14. Silva DA, DePaula RCM, Feitosa JPA, DeBrito ACF, Maciel JS, Paula HCB. Carboxymethylation of cashew tree exudate polysaccharide. Carbohydr Polym. 2004;58(2):163-71.

15. Patel M, Tekade A, Gattani S, et al. Solubility Enhancement of Lovastatin by Modified Locust Bean Gum using Solid Dispersion Techniques. AAPS Pharm Sci Tech. 2008;9(4):1262-9.

16. Biswal DR, Singh RP. Characterization of carboxymethyl cellulose and polyacrylamide graft copolymer. Carbohydr Polym. 2004;57(4):379-87.

17. Ravi PR, Ganga S, Saha RN. Design and study of lamivudine oral controlled release tablets. AAPS Pharm SciTech. 2007;8(4):E101.

18. Xu Y. Chapter 19 - Hierarchical Materials in Modern Inorganic Synthetic Chemistry, (Second Edition). 2017;545-74.

19. Roy L. Whistler. Solubility of polysaccharides and their behavior in solution, in carbohydrates in solution, Chapter 14. Advances in Chemistry. 2009;117:242 55. ISBN13: 9780841201781elSBN: 9780841222977. Published online

20. Whistler RL. Solubility of polysaccharides and their behavior in solution. Carbohydrate in Solution. Washington, DC: American Chemical Society Press. 1973; 242-55

21. Cui S, Wang Q. Understanding the conformation of polysaccharides. Food
Carbohydrates: Chemistry, Physical Properties and Applications. Boca Raton: CRC. 2005;219-61.

22. Naumann D. FT-Infrared and FT-Raman spectroscopy in biomedical research, In: Infrared and Raman Spectroscopy of Biological Materials. Marcel Dekker, Inc., NY, Basel. 2000;323-77.

23. Wiercigroch E, Szafraniec E, Czamara K, et al. Raman and infrared spectroscopy of carbohydrates: A review. Spectrochim Acta A Mol Biomol Spectrosc. 2017:185:317-35

24. Xue J, Tong S, Wang Z, Liu P. Chemical characterization and hypoglycaemic activities in vitro of two polysaccharides from Inonotus obliquus by submerged culture. Molecules. 2018;23(12):E3261.

25. Brown GD, Bauer J, Osborn HMI, Kuemmerle R. A Solution NMR Approach to Determine the Chemical Structures of Carbohydrates Using the Hydroxyl Groups as Starting Points. ACS Omega. 2018;3(12):17957-75.

26. Ren Y, Bai Y, Zhang Z, Cai W, DelRio FA. The preparation and structure analysis methods of natural polysaccharides of plants and fungi: A review of recent development. Molecules. 2019;24(17):3122.

27. Bothara SB, Singh S. Thermal studies on natural polysaccharide. Asian Pacific J Trop Biomed. 2012;2(2):S1031-5

28. Vinod VTP Sashidhar RB, Suresh KI, Rama RB, Vijaya SUVR, Prabhakar RT. Morphological, physicochemical and structural characterization of gum kondagogu (Cochlospermum gossypium): A tree gum from India. Food Hydrocoll. 2008;22(5):899-915.

29. D’Ayala GG, Malinconico M, Laurienzo P. Marine derived polysaccharides for biomedical applications: chemical modification approaches. Molecules. 2008;13(9):2069-106.

30. Ulery BD, Nair LS, Laurencin CT. Biomedical Applications of Biodegradable Polymers. J Polym Sci B Polym Phys. 2011;49(12):832-64.

31. Merkle HP. Drug delivery's quest for polymers: Where are the frontiers?. Eur J Pharm Biopharm. 2015;97:293-303.

32. Rana V, Rai P, Tiwary AK, Singh RS, Kennedy JF, Knill CJ. Modified gums: Approaches and applications in drug delivery. Carbohydr Polym. 2011;83(3):103147.

33. Chakravorty A, Barman G, Mukherjee S, Sa B. Effect of carboxymethylation on rheological and drug release characteristics of locust bean gum matrix tablets. Carbohydr Polym. 2016;144:50-8.

34. Bhatia M, Ahuja M. Psyllium arabinoxylan: Carboxymethylation, characterization and evaluation for nanoparticulate drug delivery. Int J Biol Macromol. 2015;72:495-501.

35. Paramakrishnan $\mathrm{N}$, Jha $\mathrm{S}$, Kumar KJ. Effect of carboxymethylation on physicochemical, micromeritics and release characteristics of Kyllinga nemoralis starch. Int J Biol Macromol. 2016;92:543-9.

36. Kumar A, Ahuja M. Carboxymethyl gum kondagogu: Synthesis, characterization and evaluation as mucoadhesive polymer. Carbohydr Polym. 2012;90(1):637-43.

37. Kaity S, Ghosh A. Carboxymethylation of locust bean gum: Application in interpenetrating polymer network microspheres for controlled drug delivery. Ind Engg Chem. 2013;52(30):10033-45.

38. Calderón-Castro A, Jacobo-Valenzuela N, Félix-Salazar LA, et al. Optimization of corn starch acetylation and succinylation using the extrusion process. J Food Sci Technol. 2019:56(8):3940-50.

39. Leong KH, Chung LY, Noordin MI, Mohamad K, Nishikawa M, Onuki Y, et al. Carboxymethylation of kappa-carrageenan for intestinal-targeted delivery of bioactive macromolecules. Carbohydr Polym. 2011;83(4):1507-15.

40. Tatavarti A, Mehta K, Augsburger L, Hoag S. Influence of methacrylic and acrylic acid polymers on the release performance of weakly basic drugs from sustained release hydrophilic matrices. J Pharm Sci. 2004;93(9):2319-31.

41. Gabr K. Effect of organic acids on the release patterns of weakly basic drugs from inert sustained release matrix tablets. Eur J Pharm Biopharm. 1992;38(6):199-202.

42. Siepmann J, Kranz H, Bodmeier R, Peppas NA. HPMC-matrices for controlled drug delivery: A new model combining diffusion, swelling and dissolution mechanisms and predicting the release kinetics. Pharm Res. 1999;16(11):174856

43. Siepmann J, Peppas NA. Modeling of drug release from delivery systems based on hydroxypropyl methylcellulose. Adv Drug Deliv Rev. 2001;48:139-57.

44. Siepmann J, Peppas NA. Hydrophilic matrices for controlled drug delivery: An improved mathematical model to predict the resulting drug release kinetics (the "sequential layer" model). Pharm Res. 2000;17(10):1290-8.

45. Hogan J. Hydroxypropyl methylcellulose sustained release technology. Drug Dev Ind Pharm. 1989;15(6-7):975-99.

46. Tiwari SB, Rajabi-Siahboomi AR. Extended-release oral drug delivery technologies: Monolithic matrix systems. Methods Mol Biol. Humana Press: Totowa, NJ. 2008;:217-43

47. Methocel ${ }^{\mathrm{TM}}$ Cellulose Ethers (Controlled Release). Website visited: WwW colorcon.com. 2020.

48. Yang L, Fassihi R. Examination of drug solubility, polymer types, hydrodynamics and loading dose on drug release behavior from a triple-layer asymmetric 
configuration delivery system. Int J Pharm. 1997;155(2):219-29.

49. Dabbagh MA, Ford JL, Rubinstein MH, Hogan JE, Rajabi-Siahboomi AR. Release of propranolol hydrochloride from matrix tablets containing sodium carboxymethylcellulose and hydroxypropyl methylcellulose. Pharm Dev Technol. 1999;4(3):313-24.

50. Korsmeyer RW, Gurny R, Doelker EM, Buri P, Peppas NA. Mechanism of solute release from porous hydrophilic polymers. Int J Pharm. 1983;15(1):25-35
51. Ritger PL, Peppas NA. A simple equation for describing of solute release: Fickian and non-Fickian release from non-swellable devices in the form of slabs, spheres, cylinders or discs. J Control Release. 1987;5(1):23-36.

52. Kosmidis K, Argyrakis $P$, Macheras P. Fractal kinetics in drug release from finite fractal matrices. J Chem Phys. 2003;119(12):6373-7.

53. Sasa B, Odon P, Stane S, Julijana K. Analysis of surface properties of cellulose ethers and drug release from their matrix tablets. Eur J Pharm Sci. 2006;27(4):375-83.

Article History: Submission Date : 30-07-2020; Revised Date : 18-09-2020; Acceptance Date : 12-11-2020.

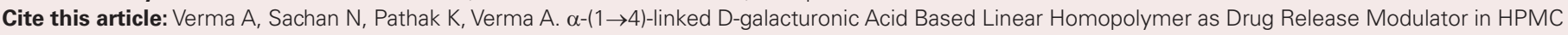
Based Hydrodynamically Balanced System. Int. J. Pharm. Investigation, 2020;10(4):492-9. 